\title{
Aspectos tomográficos e anatomopatológicos da sarcoidose pulmonar*
}

\author{
Tomographic and pathological findings in pulmonary sarcoidosis
}

Alessandro Severo Alves de Melo ${ }^{1}$, Edson Marchiori ${ }^{2}$, Domenico Capone ${ }^{3}$

Resumo Objetivo: Analisar os aspectos radiológicos observados nas tomografias computadorizadas de alta resolução de pacientes com sarcoidose e fazer a correlação com os achados anatomopatológicos. Materiais e Métodos: Foram revistos os aspectos radiológicos observados nas tomografias computadorizadas de alta resolução de dez pacientes com sarcoidose e feita correlação com material obtido de biópsias cirúrgicas ou necrópsias de quatro desses pacientes. Resultados: 0 aspecto mais frequentemente observado foi o de nódulos, com distribuição perilinfática, predominando ao longo das bainhas broncovasculares e da superfície pleural, com nódulos subpleurais e cissuras nodulares. Outros achados menos comuns foram as opacidades em vidro fosco e o espessamento de septos interlobulares. Conclusão: Em geral, todos esses achados corresponderam, anatomopatologicamente, ao acúmulo de granulomas nessas regiões.

Unitermos: Tomografia computadorizada; Sarcoidose; Anatomopatologia; Doenças pulmonares.

Abstract Objective: To analyze radiological findings observed at high-resolution computed tomography in patients with sarcoidosis, and establishing their correlation with pathological findings. Materials and Methods: High-resolution computed tomography findings in ten patients with sarcoidosis were reviewed and correlated with findings in specimens obtained by surgical biopsy or at necropsy of four of such patients. Results: The most frequently observed finding was presence of nodules with perilymphatic distribution, predominating along bronchovascular sheaths and pleural surface, with subpleural nodules and nodular scissurae. Other less frequent findings were ground-glass attenuation and interlobular septa thickening. Conclusion: In general, all the mentioned findings demonstrated anatomopathological correlation with development of granulomas in these regions.

Keywords: Computed tomography; Sarcoidosis; Pathology; Lung diseases.

Melo ASA, Marchiori E, Capone D. Aspectos tomográficos e anatomopatológicos da sarcoidose pulmonar. Radiol Bras. 2011 Jul/ Ago;44(4):220-224.

\section{INTRODUÇÃO}

A sarcoidose é uma doença multissistêmica, de etiologia desconhecida, caracterizada pela presença de inflamação granulomatosa não caseosa, que pode afetar vários sítios do organismo, com tendência a envolver o aparelho respiratório $^{(1)}$.

\footnotetext{
* Trabalho realizado no Departamento de Radiologia da Universidade Federal Fluminense (UFF), Niterói, RJ, e no Serviço de Radiodiagnóstico do Hospital Universitário Clementino Fraga Filho (HUCFF) da Universidade Federal do Rio de Janeiro (UFRJ), Rio de Janeiro, RJ, Brasil.

1. Professor Adjunto do Departamento de Radiologia da Universidade Federal Fluminense (UFF), Niterói, RJ, Brasil.

2. Professor Titular do Departamento de Radiologia da Universidade Federal Fluminense (UFF), Niterói, RJ, Coordenador Adjunto do Curso de Pós-graduação em Radiologia da Universidade Federal do Rio de Janeiro (UFRJ), Rio de Janeiro, RJ, Brasil.

3. Professor Assistente de Pneumologia da Universidade do Estado do Rio de Janeiro (UERJ), Rio de Janeiro, RJ, Brasil.

Endereço para correspondência: Dr. Edson Marchiori. Rua Thomaz Cameron, 438, Valparaíso. Petrópolis, RJ, Brasil, 25685-120. E-mail: edmarchiori@gmail.com

Recebido para publicação em 17/3/2011. Aceito, após revisão, em 20/5/2011.
}

Embora seja, normalmente, doença autolimitada para a maioria dos pacientes, que curam ou estabilizam sem o uso de imunossupressores ou agentes anti-inflamatórios, pode haver o desenvolvimento de fibrose pulmonar extensa e irreversível, que leva à deterioração progressiva da função respiratória ${ }^{(\mathbf{1})}$.

Uma análise adequada de qualquer imagem radiológica requer compreensão da anatomia normal e das alterações que ocorrem com a doença. Correlação anatomorradiológica é essencial para o entendimento das bases anatômicas da formação da imagem ${ }^{(2)}$.

Muitos trabalhos têm descrito os aspectos da sarcoidose na tomografia computadorizada; contudo, apenas um pequeno número de estudos correlacionou os achados da tomografia computadorizada de alta resolução (TCAR) com a anatomia patológica ${ }^{(2-4)}$. Esta correlação, por vezes, se torna difícil, uma vez que os pacientes que morrem em geral são aqueles com extensas lesões fibróticas pulmonares, e biópsia a céu aberto raramente é feita na sarcoidose ${ }^{(\mathbf{5})}$.

No presente trabalho são discutidos os principais aspectos da sarcoidose observados na TCAR e é feita correlação com os aspectos anatomopatológicos encontrados.

\section{MATERIAIS E MÉTODOS}

Foram revistos os aspectos radiológicos observados nas TCARs de dez pacientes com sarcoidose, estudados em instituições hospitalares universitárias do Rio de Janeiro, confirmados histologicamente, e feita correlação com material obtido de biópsias cirúrgicas ou necrópsias de quatro desses pacientes. A faixa etária variou de 30 a 68 anos, com média de 48 anos, sendo sete pacientes do sexo feminino e três do sexo masculino. 


\section{RESULTADOS}

$\mathrm{O}$ aspecto mais frequentemente observado foi o de nódulos, com distribuição perilinfática. As lesões predominaram ao longo das bainhas broncovasculares e da superfície pleural, com nódulos subpleurais e cissuras nodulares (Figura 1). Espessamento de septos interlobulares foi achado pouco frequente. Outro padrão encontrado foi o de vidro fosco, em geral associado a pequenos nódulos (Fi- gura 2). Em geral, todos esses achados corresponderam, anatomopatologicamente, ao acúmulo de granulomas nessas regiões. Outro aspecto interessante observado foi o de aprisionamento aéreo, determinado por estreitamento da luz

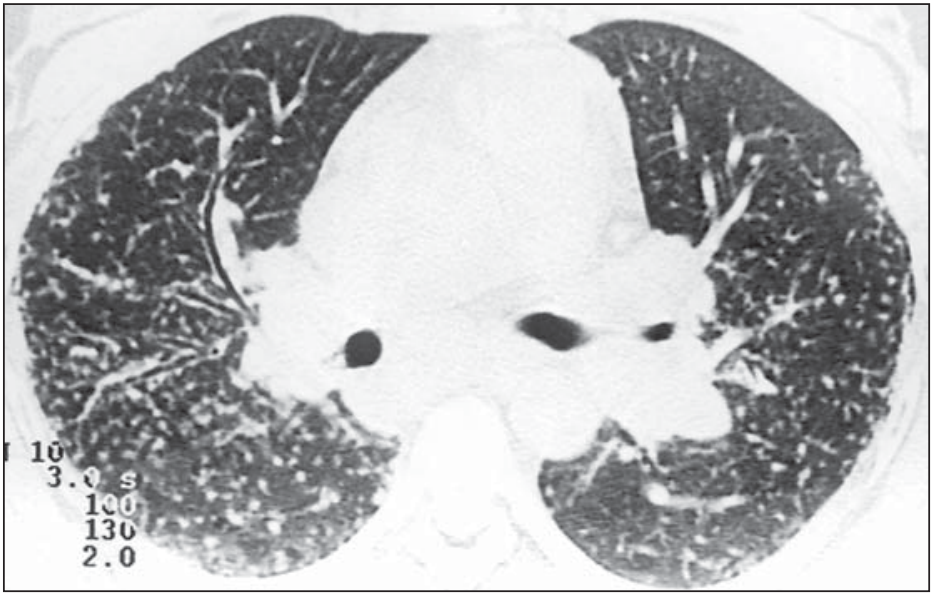

A

Figura 1. Três pacientes diferentes. Em A e B observam-se espessamento das bainhas broncovasculares e acúmulo de nódulos na região subpleural. Em $\mathbf{B}$ e $\mathbf{C}$ chama a atenção, também, o aspecto das cissuras nodulares. Observar, em $\mathbf{A}$, linfonodomegalias hilares, e em $\mathbf{C}$, aspecto de vasos com calibre aparentemente aumentado, especialmente à direita, determinado por espessamento da bainha perivascular. Em $\mathbf{D}$ e E, cortes histológicos mostram o acúmulo de granulomas na região subpleural (D) e ao longo do septo interlobular (E), responsável pelo aspecto nodular destas estruturas na TCAR.

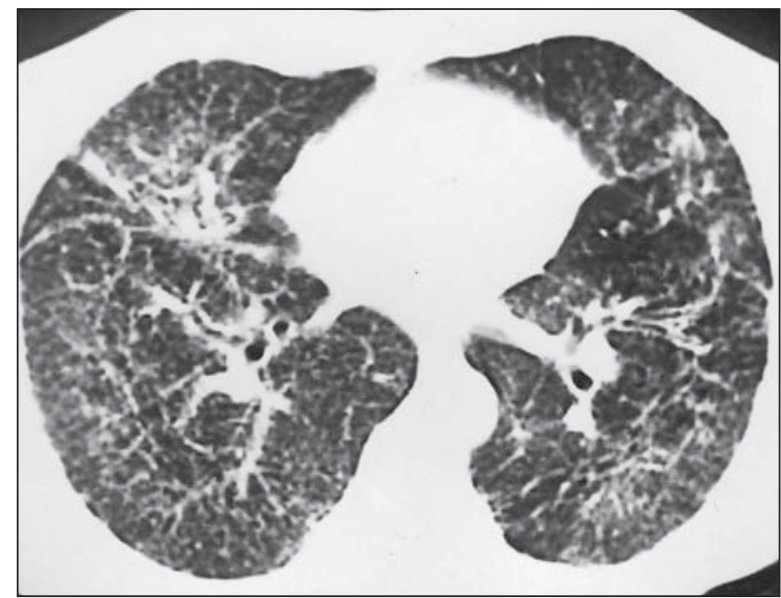

B

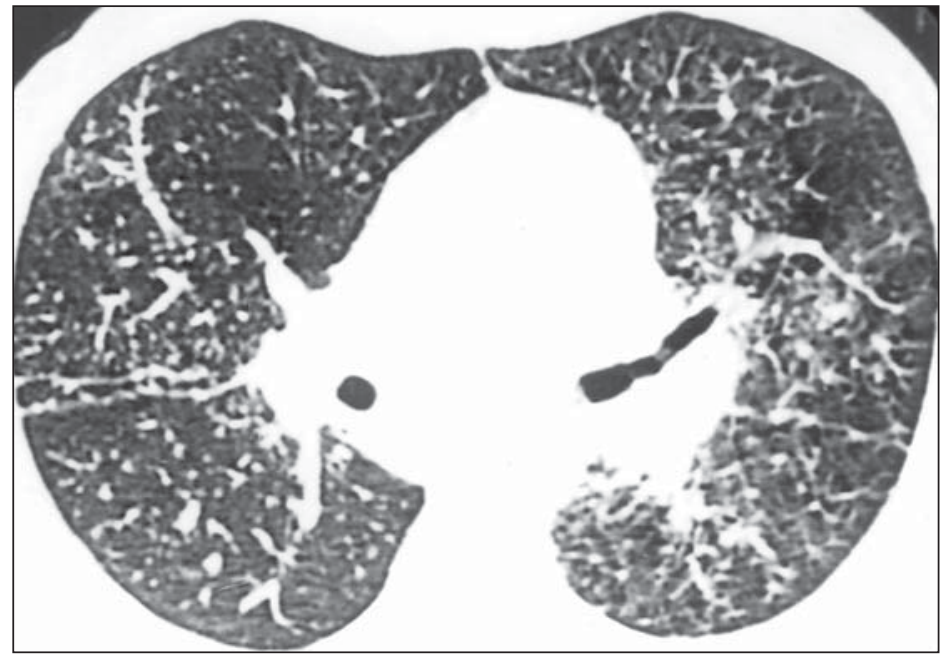

C

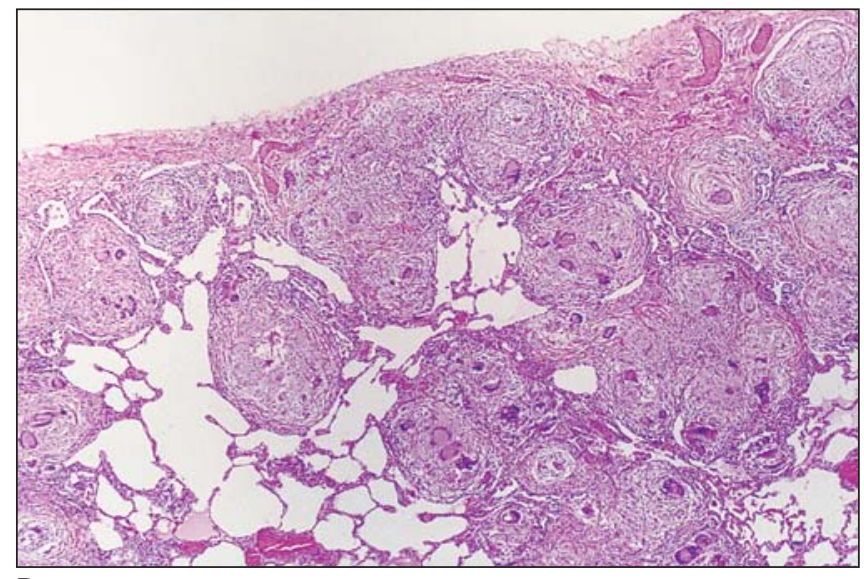

D

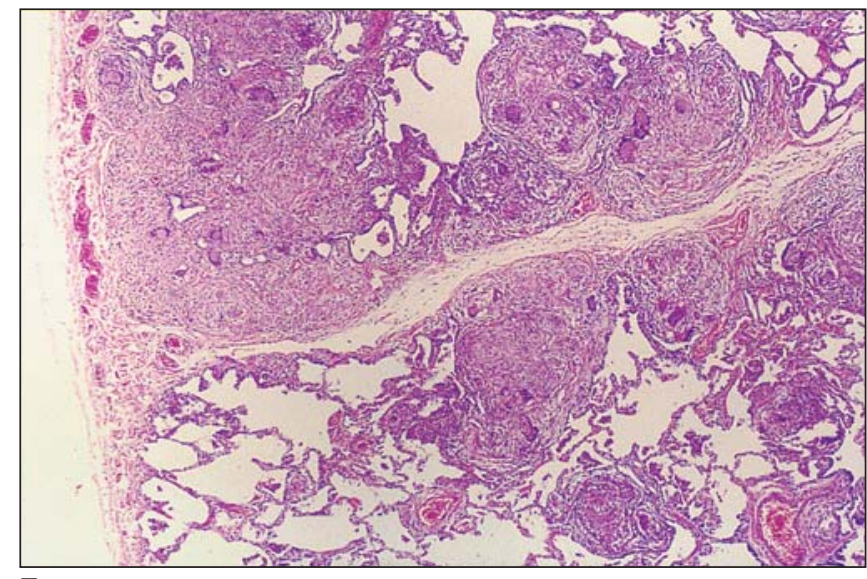

E 


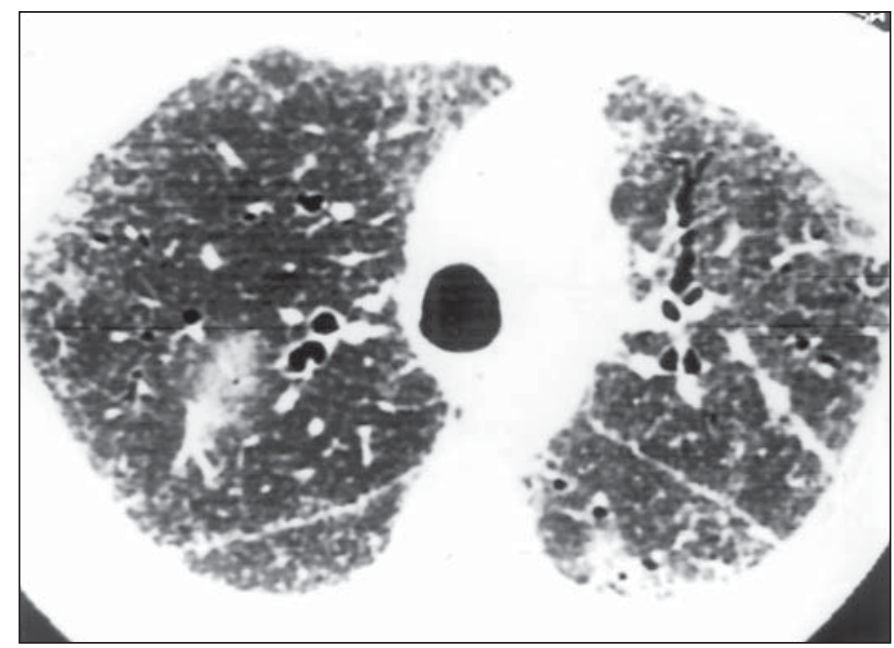

A

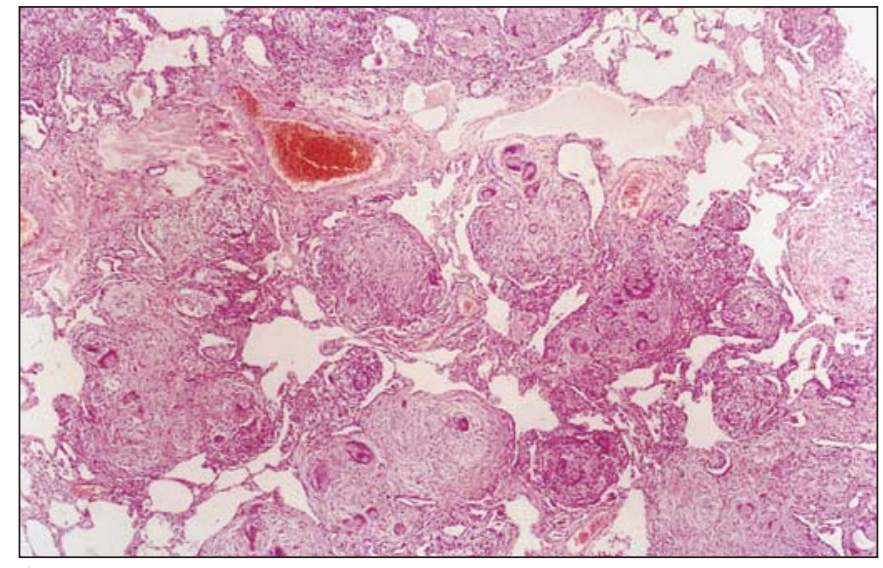

C

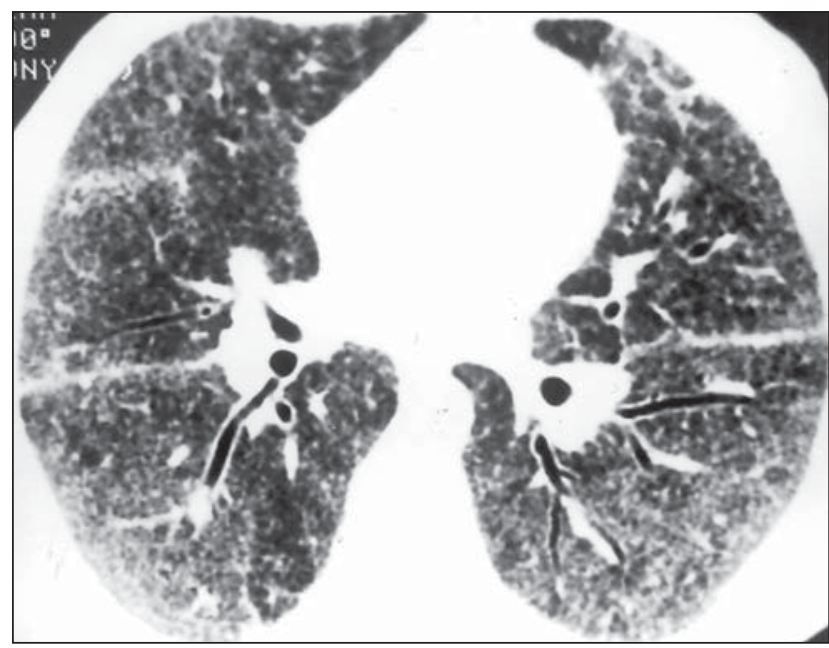

B

Figura 2. Em A e B, TCAR evidenciando padrão em vidro fosco, com aspecto de micronódulos difusos, e cissura nodular. Observar, também, espessamento irregular das bainhas peribroncovasculares. Em C, corte histológico demonstrando que as opacidades em vidro fosco se deviam a acúmulo de granulomas esparsos pelo parênquima, comprimindo os espaços alveolares. brônquica por granulomas peribrônquicos (Figura 3).

\section{DISCUSSÃO}

O achado histopatológico básico na sarcoidose é o granuloma. Os granulomas não caseosos são confinados ao compartimento intersticial dos pulmões e mostram uma distribuição perilinfática, ao longo das bainhas broncovasculares, septos interlobulares e superfície pleural ${ }^{(4,6,7)}$, incluindo a pleura cissural ${ }^{(7)}$. Esta preferência pelas regiões peribroncovasculares explica a alta frequência de biópsias transbrônquicas positivas ${ }^{(8)}$. A doença, de maneira geral, predomina nos campos superiores e médios e nas regiões posteriores ${ }^{(8)}$. $\mathrm{Na}$ verdade, os granulomas podem produzir três padrões: nódulos, espessamento irregular das estruturas e vidro fosco ${ }^{(2)}$. Também áreas de consolidação podem ser vistas, por confluência irregular dos nódulos $(8)$.

Os nódulos são a anormalidade mais frequentemente vista na $\operatorname{TCAR}^{(7)}$. Eles apresentam, em geral, diâmetro de 2 a 10 $\mathrm{mm}$, margens irregulares ${ }^{(3,7)}$, e representam, anatomopatologicamente, agregados de granulomas ${ }^{(\mathbf{3}, 6,7)}$.

Os conglomerados de granulomas podem também, menos frequentemente, originar grandes nódulos (maiores que $1 \mathrm{~cm}$ ) e grandes opacidades, de contornos mal definidos $^{(2,3,5,7,8)}$.

Os pequenos granulomas individualizados, esparsos pelo parênquima, dão o padrão em vidro fosco ${ }^{(7)}$. Eles não têm dimensão suficiente para serem individualizados na TCAR, mas produzem aumento difuso da atenuação pulmonar, originando este padrão ${ }^{(6)}$. Inicialmente, alguns autores sugeriram que este padrão se devia à alveolite, mas estudos poste- riores demonstraram que, de regra, nas fases agudas da sarcoidose a alteração era determinada apenas por granulomas ${ }^{(2,3,7)}$. Ainda que o padrão em vidro fosco usualmente reflita a presença de doença potencialmente tratável ou reversível, em alguns pacientes ela resulta de fibrose ${ }^{(1,7)}$. A presença de fibrose é sugerida pela concomitância de bronquiectasias de tração, bronquiolectasias e distorção parenquimatosa $^{(\mathbf{1})}$.

Embora os granulomas apresentem distribuição perilinfática, na sarcoidose eles ocorrem em maior número ao longo das bainhas broncovasculares e em menor quantidade nos septos interlobulares e nas regiões subpleurais ${ }^{(3)}$. O espessamento de septos interlobulares é achado pouco frequente na sarcoidose ${ }^{(7,9)}$.

Opacidades lineares são menos frequentes e menos profusas que nódulos. Estruturas poligonais, como as vistas na 


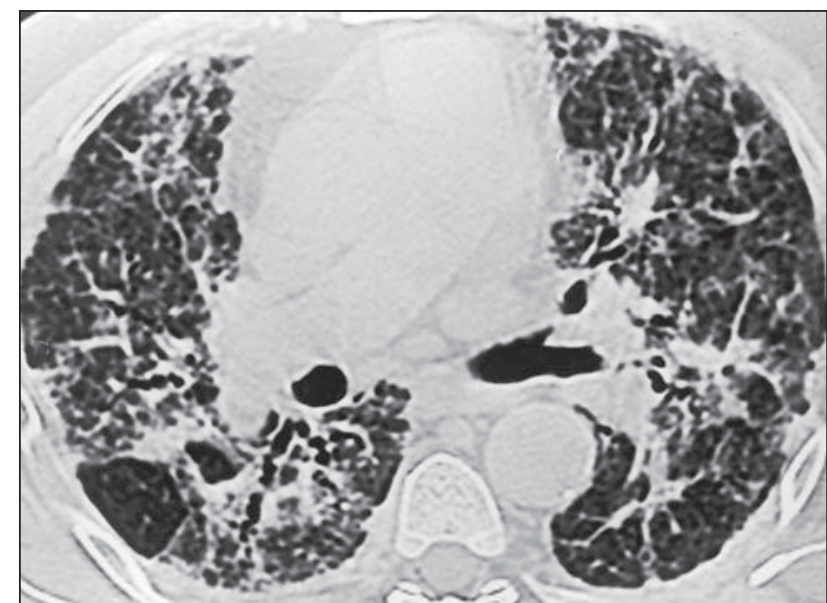

A

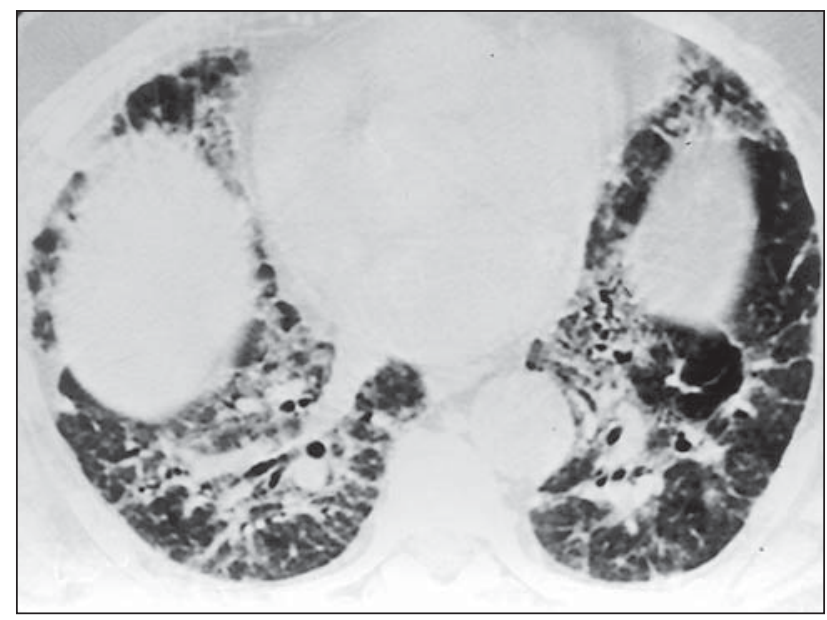

C

Figura 3. Em A, TCAR demonstrando, além de septos espessados com evidências de fibrose, lóbulo pulmonar secundário inflado no lobo inferior direito. Em B, corte evidenciando granuloma comprimindo bronquíolo e determinando aprisionamento aéreo. Em C, TCAR mostrando lóbulo pulmonar inflado na base do pulmão esquerdo, com estrutura centrolobular em forma de arco, de contornos nodulares. Em $\mathbf{D}$, corte da região centrolobular mostrando múltiplos granulomas na bainha peribroncovascular, estreitando a luz bronquiolar, explicando o enfisema valvular e a configuração centrolobular em arco, com nodulações, observados em C.

linfangite carcinomatosa, são achados muito raros na sarcoidose ${ }^{(7,9)}$. Na sarcoidose, os nódulos têm limites irregulares; na linfangite, em geral, são mais $\operatorname{lisos}^{(\mathbf{9})}$. Por vezes, há dificuldade no reconhecimento histológico da distribuição linfática, pelo envolvimento desproporcional de um sítio anatômico em relação a outro. $\mathrm{Na}$ sarcoidose há envolvimento muito maior das bainhas broncovasculares, com pouco envolvimento dos septos e da superfície pleural, eventualmente sugerindo mais um padrão bronquiolocêntrico/angiocêntrico do que propriamente um padrão linfático ${ }^{(4)}$.

Os granulomas proliferando ao longo das bainhas broncovasculares provocam seu espessamento irregular ${ }^{(3)}$, com as paredes das artérias e brônquios assumindo

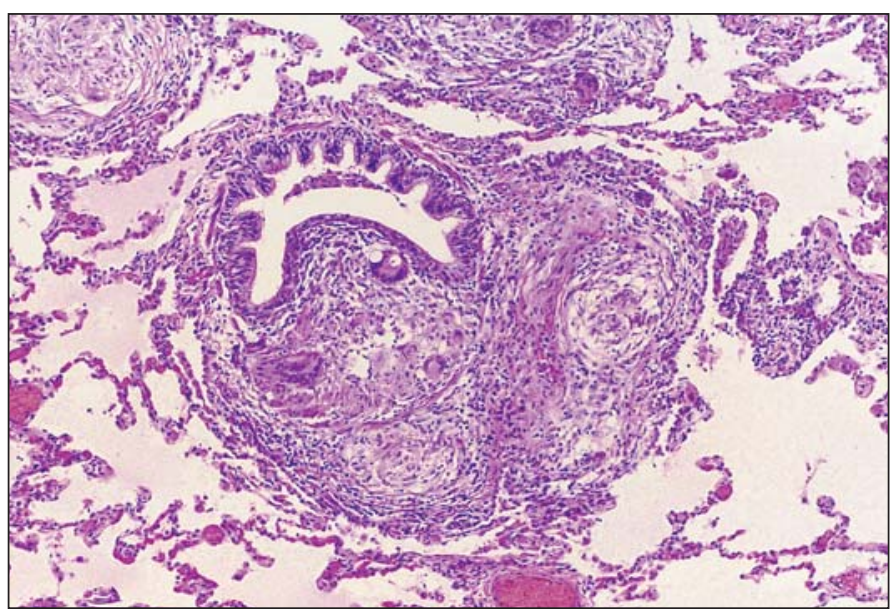

B

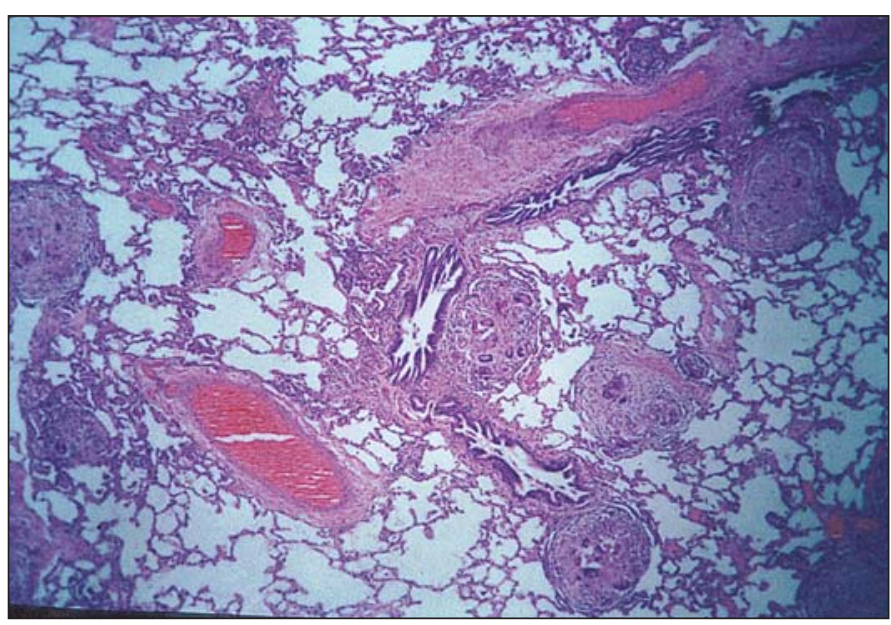

D aspecto "em rosário", pela nodularidade dos seus contornos ${ }^{(7)}$. Também as cissuras e os septos interlobulares podem apresentar este aspecto "em rosário" (7).

A superfície pleural pode ter aspecto nodular; na TCAR, os granulomas formados na pleura não podem ser diferenciados daqueles formados em septos alveolares adjacentes à pleura ${ }^{(3)}$.

É importante reconhecer que os elementos celulares e granulomatosos de inflamação indicam atividade, reversibilidade e resposta favorável ao tratamento, enquanto o comprometimento fibrótico indica irreversibilidade e resposta pobre à terapêutica ${ }^{(\mathbf{1})}$.

De modo geral, as alterações determinadas pelos granulomas (pequenos nódulos, grandes nódulos, vidro fosco e espessamento nodular - "em rosário" de estruturas intersticiais) são reversíveis $^{(\mathbf{3 , 5})}$, uma vez que representam lesões inflamatórias ativas ${ }^{(5)}$. Com ou sem tratamento, estas lesões podem permanecer por meses ou anos ou serem reabsorvi$\operatorname{das}^{(1,8)}$.

No entanto, alguns achados são irreversíveis, representando manifestações de fibrose. Um aspecto comum na fibrose é a distorção arquitetural, que pode causar rechaço posterior do brônquio principal ou dos brônquios superiores ${ }^{(5,7,8)}$. Outros achados são as bronquiectasias de tração, o faveolamento ${ }^{(\mathbf{5 , 7 , 8 )}}$, os cistos e bolhas $^{(7)}$ e o aparecimento de linhas parenquimatosas, septais e não septais ${ }^{(\mathbf{5})}$. Podem aparecer opacidades lineares longas e irregulares, principalmente localiza- 
das ao longo de bainhas broncovasculares, irreversíveis, também como manifestação precoce de fibrose ${ }^{(7)}$. Aspecto de interfaces irregulares, semelhante à da fibrose pulmonar idiopática, também foi descrito, determinado por fibrose septal localizada ${ }^{(8)}$. A fibrose pode ser tão grande, a ponto de formar massas peri-hilares, lembrando a fibrose maciça progressiva da silicose ${ }^{(7)}$. Outra possibilidade é a persistência de padrão em vidro fosco após o tratamento, que é atribuído à fibrose de septos alveolares ${ }^{(5)}$.

No que se refere à função pulmonar, estudos têm mostrado que a profusão de granulomas em biópsias não se correlaciona com a deterioração da função ${ }^{(\mathbf{1})}$. Parece que a alteração funcional está mais relacionada à localização estratégica de alguns granulomas (envolvendo bronquíolos e causando aumento da resistência ao fluxo aéreo) do que à sua quantidade $^{(\mathbf{1 , 6})}$. O estreitamento da via aérea determinado por granulomas peribrônquicos resulta em aprisionamento de ar na TCAR, especialmente nos exames feitos em fase expiratória, em que o padrão em mosaico pode ser visto ${ }^{(6)}$.

\section{REFERÊNCIAS}

1. Remy-Jardin M, Giraud F, Remy J, et al. Pulmonary sarcoidosis: role of CT in the evaluation of disease activity and functional impairment and in prognosis assessment. Radiology. 1994;191:67580.

2. Müller NL, Miller RR. Ground-glass attenuation, nodules, alveolitis, and sarcoid granulomas. Radiology. 1993;189:31-2.
3. Nishimura K, Itoh H, Kitaichi M, et al. Pulmonary sarcoidosis: correlation of CT and histopathologic findings. Radiology. 1993;189:105-9.

4. Colby TV, Swensen SJ. Anatomic distribution and histopathologic patterns in diffuse lung disease: correlation with HRCT. J Thorac Imaging. 1996;11:126.

5. Brauner MW, Lenoir S, Grenier P, et al. Pulmonary sarcoidosis: CT assessment of lesion reversibility. Radiology. 1992;182:349-54.

6. Gleeson FV, Traill ZC, Hansell DM. Evidence on expiratory CT scans of small-airway obstruction in sarcoidosis. AJR Am J Roentgenol. 1996;166: 1052-4.

7. Traill ZC, Maskell GF, Gleeson FV. High-resolution CT findings of pulmonary sarcoidosis. AJR Am J Roentgenol. 1997;168:1557-60.

8. Brauner MW, Grenier P, Mompoint D, et al. Pulmonary sarcoidosis: evaluation with high-resolution CT. Radiology. 1989;172:467-71.

9. Müller NL, Kullnig P, Miller RR. The CT findings of pulmonary sarcoidosis: analysis of 25 patients. AJR Am J Roentgenol. 1989;152:1179-82. 